\title{
BREAST CANCER
}

\section{Stable breast cancer xenograft models}

A new study has demonstrated stable, transplantable, xenograft models that are biologically consistent with the breast tumours of origin.

A factor that hinders breast cancer research is the limited number of in vivo models that accurately reflect the heterogeneity of human tumours. In vivo therapeutic studies often use xenograft models in which human cancer cell lines are transplanted into mice. However, these cell lines have been shown to evolve in culture, acquiring genomic changes not present in the original tumour.

Researchers propagated human breast tumours grown in the epithelium-free mammary fat pad of immunocompromised mice with oestradiol supplementation to increase engraftment rate. The resulting xenografts were stable, even over several years of continuous passage by serial transplantation into new host mice. Importantly, as lead investigator
Michael T. Lewis explains, "the response of xenografts to treatment was qualitatively identical in most cases to the clinical response of the tumour of origin ... had they not responded similarly, their utility in preclinical studies would be much more limited than desired."

Lewis emphasizes the usefulness of these xenografts for future research: “....if our collection is combined with other similar collections, the breast cancer community now has access to a statistically robust number of preclinical models representing each major clinically-defined breast cancer subtype in which to test novel therapeutics efficiently, and in a cost-effective manner, without risk to patients."

Vanessa Marchesi

Original article Zhang, $X$. et al. A renewable tissue resource of phenotypically stable, biologically and ethnically diverse, patient-derived human breast cancer xenografts. Cancer Res. doi:10.1158/0008-5472.CAN-12-4081 